\title{
Reading development in the digital age
}

DOI: $10.37716 /$ HBAB.2020010402

\author{
Li Hai $\operatorname{Tan}^{1,2 *}, \operatorname{Min} \mathrm{Xu}^{2,3 *}$
}

The rapid development of digital technology has led to massive changes in the way we communicate and the way we read. In contrast to the focused, uninterrupted and time-consuming cognitive processes that are vital for successful reading, digital technology greatly emphasizes on rapid and massive information processing. Accumulated evidence has suggested that the reliance on digital media makes reading shallower, more fragmented and less attentive. Digital technology has an even more profound impact on Chinese reading development, with brain and behavioral evidence showing that increasing dependence on electronic modes is associated with weaker cortical activations and a marked decrease in reading ability. Here we provide an overview of the impact of digital technology on reading development, and urge caution in the rush to a complete transition to digital learning while abandoning classic methods of learning, such as handwriting.

Keywords: reading development, digital technology, brain, Chinese reading, handwriting

Written language - a recent cultural invention that emerged about five thousand years ago - plays a critical role in knowledge spread and continuity of human civilization. The ability to read or make sense of written symbols therefore serves as an important portal to knowledge. Reading, a crucial skill for children to master, is not only necessary for academic success, but is also important for maintaining a decent quality of life in our increasingly literate society.

In the digital age, students are currently immersed in information technology and future generations will continue to do so, leading to massive changes in the way we communicate and the way we read. For instance, electronic forms of communication via computers, cell phones, and other devices make digitized books accessible with superior flexibility, allowing education to move from the traditional paper-and-pencil method to a digital approach. While rapidlyevolving digital technology makes a learning revolution possible, it comes with some serious risks and side effects. Increasing research has been performed to examine the impact of digital technology usage on memory (1), attention and cognitive control (2), motor skills (3), and so on. Digital technology greatly emphasizes on rapid and massive information processing in quick and interactive modes of communication, which is in sharp contrast to the focused, uninterrupted and time-consuming cognitive processes that are vital for successful reading. Its impact on reading development has thus attracted considerable attention (4-7), raising a concern that the advent of digital media makes reading shallower, more fragmented, and less attentive. Frequent exposure to digital technology, while it affects adults too, has been reported to influence children more profoundly. As children's reading ability develops through explicit training, their highly plastic brains can more easily be habituated to the environmental stimuli ( 8$)$.

Research indicates that reading electronic books is less efficient than reading traditional print books (9-11), though electronic texts have many advantages such as easy updates, quick sharing, and embedded with multimedia and interactive content. For example, built-in hyperlinks in the text have been found to impair reading performance, probably due to increased demands of decisionmaking and visual processing (12). In addition, readers who click on too many hyperlinks can easily be distracted and lose track of

${ }^{1}$ Guangdong-Hongkong-Macau Institute of CNS Regeneration and Ministry of Education CNS Regeneration Collaborative Joint Laboratory, Jinan University, 510632, Guangzhou, China. ${ }^{2}$ Center for Language and Brain, Shenzhen Institute of Neuroscience, 518057, Shenzhen, China. ${ }^{3}$ Center for Brain Disorders and Cognitive Sciences, Shenzhen University, 518060, Shenzhen, China.

*Corresponding authors. Email: tanlh@sions.cn (L.H.T.), xumin@szu.edu.cn (M.X.) the text (13). Moreover, readers with weak working memory and weedy prior knowledge are particularly susceptible to the negative influence of digital reading $(12,13)$, as they have fewer available cognitive resources for reading comprehension.

Furthermore, with multiple streams of content available from electronic devices (e.g., web surfing, text messaging, music, video, and so on), children who read using computers and cell phones become increasingly engaged in multitasking behaviors, which are consistently linked to increased distractibility and poorer academic performance (14). Ophir et al. reported negative impact of media multitasking on cognitive control; in particular, heavy media multitaskers showed reduced ability to filter interference from irrelevant environmental stimuli (2). The observed decreased cognitive control performance in the heavy media multitaskers was associated with reduced gray matter density in the anterior cingulate cortex (15), a region critical for cognitive control and conflict processing. The distraction and interruption introduced by digital reading increase the complexity and difficulty of reading, and children, whose attention systems and executive functions are immature, are especially at risk.

The impact of digital technology is even more profound on Chinese reading development. It has been demonstrated that increasing reliance on electronic modes over handwriting is accompanied by a marked decrease in reading ability of Chinese children (7). In a sample of nearly 6000 primary school Chinese children, the overall incidence rate of children with severe reading difficulty, defined using the same criteria as 15 and 30 years ago, is $28 \%$, which is much higher than ever reported. Further, children's reading performance significantly decreases with their utilization of the pinyin (romanization) input method and electronic devices, but increases with their time spent on handwriting (7). This finding is corroborated by He et al.'s study, which showed that electronic device usage significantly increases the risk of Chinese dyslexia in a sample of 5063 primary school children (16).

A recent fMRI study by Zhou et al. further demonstrated the impact of pinyin typing on neurodevelopment of reading in Chinese school-aged children (17). They found that more frequent usage of pinyin typing and digital devices in children was associated with overall weaker cortical activations, weaker functional connections, and reduced gray matter volume within the reading cortical network, suggesting that reliance on typewriting over handwriting may interfere with children's neurodevelopment of reading. It should also be noted that children are not the only ones who are negatively affected by excessive daily usage of electronic devices. Recent studies showed that frequent usage of electronic 
devices in adults led to reduced or inefficient coordination of cognitive resources during reading at both behavioral (18) and neurocognitive levels (19). Hsu et al.'s fMRI study showed that adults who frequently used electronic devices had lower activity in the left inferior frontal gyrus and left insula during reading - regions that play an important role in language processing and attention switching (19).

In written Chinese, graphic units (characters) map onto morphemes and syllables, rather than phonemes. The challenging properties of Chinese characters (visually complex forms without phonemic constituents) lead to a prevalent strategy for learning to read by repeatedly writing/copying the characters $(20,21)$. Through writing, children learn how to deconstruct characters into strokes and stroke patterns, then regroup these stroke patterns into a square unit, and with practice, to establish long-term motor memory of Chinese character writing. Research has consistently demonstrated the importance of handwriting in Chinese reading development ( 7 , 20,22). On the other hand, the pinyin input method, which is used by more than $97 \%$ of computer users in mainland China, does not require the users to construct the characters by combining strokes, as one would do in writing characters by hand. The pinyin input method allows users to input a Chinese character by typing its pinyin spelling, a phonetic transcription of the character's pronunciation, and then selecting the desired character from a list of characters sharing the same pinyin spelling (see Fig. 1). Visuospatial properties of characters indispensable to Chinese reading are never involved during the typing process, and thus this typing method seems to interfere with the learning of the graphical representation of Chinese characters during reading acquisition. Indeed, it has been found that people who used pronunciationbased input methods (e.g., pinyin input method) performed poorer in reading ability tests than people who used orthographic-based input methods (e.g., stroke-based input method or handwriting on electronic devices) (23).

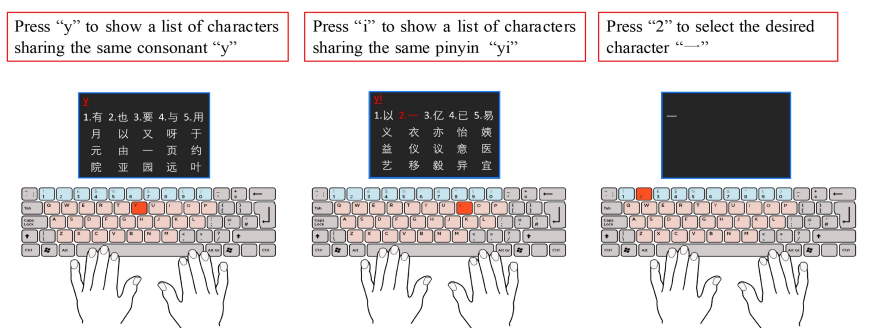

Fig. 1. An example of using the pinyin input method to type the Chinese character "-" (pinyin "yi", meaning "one").

The Chinese language has survived technological challenges of modernization in the digital age (24), but the benefits of electronic communicating may come at a cost in terms of proficient learning of written Chinese. To counteract the problem of severe decline in reading and writing abilities among Chinese students in recent years, the Chinese government has initiated a series of educational policies to encourage more handwriting and to put more emphasis on studying Chinese relative to studying English which is a popular second language in China. Other measures include instructing primary schools to increase writing classes and increasing the weighting given to the Chinese language test in the National College Entrance Examination. The age at which pinyin is introduced in primary school has also been postponed, which to some extent reduces the negative effect of pinyin on Chinese reading acquisition. Moreover, there have also been television programs focusing on preserving and promoting Chinese characters, such as the Chinese Characters Dictation Contest aired on China Central Television (CCTV) during prime time. These programs have reinvigorated the enthusiasm for writing characters among Chinese people.

Chinese reading is not the only skill that benefits from handwriting. Previous studies demonstrated that the effect of learning letter stimuli through handwriting is better than learning through typing $(25,26)$. In a neuroimaging study, Nakamura et al. reported a universal writing-on-reading effect across languages, showing that reading handwritten words activated a left frontal pre-motor area that is involved in handwriting in both Chinese and French readers (27). This finding suggests that handwriting is a powerful procedure for establishing high quality representation of written word form knowledge $(28,29)$.

The declining reading and writing abilities, alongside the increasing reliance on digital devices and the internet, may accelerate the rate of language endangerment and language death. Due to the limited number of languages currently supported by existing digital devices and the internet, there is a very poor online linguistic diversity. According to the 2015 Broadband Progress Report published by the United Nation Broadband Commission, only $5 \%$ of the world's languages are represented on the internet, and the internet's content is dominated by a few major languages (e.g., $55.2 \%$ of the 10 million most popular websites are in English) (30). As a result, digital communication in modern society compels people to learn and communicate using the dominant language online, whereas fewer and fewer people would read and write the languages that do not have a digital future.

The digital age is opening new opportunities for richer learning environment for children, but it is also challenging their development of reading and writing abilities. We urge caution in the rush to a complete transition to digital learning and digital education while abandoning the classic methods of learning, such as handwriting. To meet the grand challenges, action must be taken to balance the use of digital technology with the traditional critical factors for human development.

\section{REFERENCES}

1. B. Sparrow, J. Liu, D. M. Wegner, Google effects on memory: Cognitive consequences of having information at our fingertips. Science 333, 776-778 (2011).

2. E. Ophir, C. Nass, A. D. Wagner, Cognitive control in media multitaskers. Proc. Natl. Acad. Sci. U.S.A. 106, 15583-15587 (2009).

3. K. Wei, X. Yan, G. Kong, C. Yin, F. Zhang, Q. Wang, K. P. Kording, Computer use changes generalization of movement learning. Curr. Biol. 24, 82-85 (2014).

4. N. G. Carr, The Shallows: What the Internet Is Doing to Our Brains (W.W. Norton, New York, 2010).

5. M. Wolf, C. Ullman-Shade, S. Gottwald, The emerging, evolving reading brain in a digital culture: Implications for new readers, children with reading difficulties, and children without schools. J. Cogn. Educ. Psychol. 11, 230-240 (2012).

6. K. K. Loh, R. Kanai, How has the internet reshaped human cognition? Neuroscientist 22, 506520 (2016).

7. L. H. Tan, M. Xu, C. Q. Chang, W. T. Siok, China's language input system in the digital age affects children's reading development. Proc. Natl. Acad. Sci. U.S.A. 110, 1119-1123 (2013).

8. S. E. Fox, P. Levitt, C. A. Nelson, How the timing and quality of early experiences influence the development of brain architecture. Child Dev. 81, 28-40 (2010).

9. D. B. Daniel, W. D. Woody, E-textbooks at what cost? Performance and use of electronic v. print texts. Comp. Educ. 62, 18-23 (2013).

10. D. B. Daniel, D. T. Willingham, Electronic textbooks: Why the rush? Science 335, 1569-1571 (2012).

11. Z. Liu, Reading behavior in the digital environment: Changes in reading behavior over the past ten years. J. Doc. 61, 700-712 (2005).

12. D. DeStefano, J.-A. LeFevre, Cognitive load in hypertext reading: A review. Comput. Human Behav. 23, 1616-1641 (2007).

13. J. L. Plass, D. M. Chun, R. E. Mayer, D. Leutner, Cognitive load in reading a foreign language text with multimedia aids and the influence of verbal and spatial abilities. Comput. Human Behav. 19, 221-243 (2003).

14. L. M. Carrier, L. D. Rosen, N. A. Cheever, A. F. Lim, Causes, effects, and practicalities of everyday multitasking. Dev. Rev. 35, 64-78 (2015).

15. K. K. Loh, R. Kanai, Higher media multi-tasking activity Is associated with smaller gray-matter 
density in the anterior cingulate cortex. PLOS ONE 9, e106698 (2014).

16. Z. He, S. Shao, J. Zhou, J. Ke, R. Kong, S. Guo, J. Zhang, R. Song, Does long time spending on the electronic devices affect the reading abilities? A cross-sectional study among Chinese school-aged children. Res. Dev. Disabil. 35, 3645-3654 (2014).

17. W. Zhou, V. P. Y. Kwok, M. Su, J. Luo, L. H. Tan, Children's neurodevelopment of reading is affected by China's language input system in the information era. npj Science of Learning $\mathbf{5}$ 3 (2020).

18. D. J. Follmer, S.-Y. Fang, R. B. Clariana, B. J. F. Meyer, P. Li, What predicts adult readers' understanding of STEM texts? Read. Writ. 31, 185-214 (2018).

19. C.-T. Hsu, R. B. Clariana, B. Schloss, P. Li, Neurocognitive signatures of naturalistic reading of scientific texts: A fixation-related fMRI study. Sci. Rep. 9, 10678 (2019).

20. L. H. Tan, J. A. Spinks, G. F. Eden, C. A. Perfetti, W. T. Siok, Reading depends on writing, in Chinese. Proc. Natl. Acad. Sci. U.S.A. 102, 8781-8785 (2005).

21. C. Leong, Cognition, Intelligence, and Achievement: A Tribute to JP Das (Elsevier Academic Press, San Diego, 2015).

22. R. V. Y. Tso, T. K. Au, J. H. Hsiao, Writing facilitates learning to read in Chinese through reduction of holistic processing: A developmental study. J. Vision 12, 530 (2012).

23. W. T. Siok, C. Y. Liu, Differential impacts of different keyboard inputting methods on reading and writing skills. Sci. Rep. 8, 1-13 (2018).

24. A. L. Mackay, Character-building. Nature 410, 19 (2001).

25. M. Longcamp, C. Boucard, J.-C. Gilhodes, J.-L. Anton, M. Roth, B. Nazarian, J.-L. Velay, Learning through hand- or typewriting influences visual recognition of new graphic shapes: Behavioral and functional imaging evidence. J. Cogn. Neurosci. 20, 802-815 (2008).
26. M. Longcamp, M.-T. Zerbato-Poudou, J.-L. Velay, The influence of writing practice on letter recognition in preschool children: A comparison between handwriting and typing. Acto Psychol. (Amst.) 119, 67-79 (2005).

27. K. Nakamura, W. J. Kuo, F. Pegado, L. Cohen, O. J. L. Tzeng, S. Dehaene, Universal brain systems for recognizing word shapes and handwriting gestures during reading. Proc. Natl. Acad. Sci. U.S.A 109, 20762-20767 (2012).

28. C. A. Perfetti, L. H. Tan, Write to read: The brain's universal reading and writing network. Trends in Cogn. Sci. 17, 56-57 (2013).

29. F. Cao, M. Vu, D. H. L. Chan, J. M. Lawrence, L. N. Harris, Q. Guan, Y. Xu, C. A. Perfetti, Writing affects the brain network of reading in Chinese: A functional magnetic resonance imaging study. Hum. Brain Mapp. 34, 1670-1684 (2013).

30. U. N. B. Commission, The State of Broadband 2015 (2015).

Acknowledgments: Funding: This work was supported by Shenzhen Basic Research Grants (JCYJ20170818110103216, JCYJ20170412164259361, 2019SHIBS0003, JCYJ20170412164413575), Shenzhen Peacock Team Plan (KQTD2015033016104926), Guangdong Pearl River Talents Plan Innovative and Entrepreneurial Team Grant (2016ZT06S220), Guangdong Key Basic Research Grant (2018B030332001), and The National Natural Science Foundation of China (31700951). Author contributions: L.H.T and M.X. conceived the idea and wrote the manuscript. Competing interests: The author declares no competing interests, financial or otherwise, in completion of this work. Data and materials availability: All data and materials used in the preparation of this paper are cited herein. Additional information related to this paper may be requested from the corresponding author. 